\title{
Quantum dot asymmetry and the nature of excited hole states probed by the doubly positively charged exciton $X^{2+}$
}

\author{
Daniel Dufåker, K. Fredrik Karlsson, L. O. Mereni, V. Dimastrodonato, G. Juska, E. Pelucchi
} and Per-Olof Holtz

\section{Linköping University Post Print}

\section{Tweet}

N.B.: When citing this work, cite the original article.

Original Publication:

Daniel Dufåker, K. Fredrik Karlsson, L. O. Mereni, V. Dimastrodonato, G. Juska, E. Pelucchi and Per-Olof Holtz, Quantum dot asymmetry and the nature of excited hole states probed by the doubly positively charged exciton $X^{2+}, 2013$, Physical Review B. Condensed Matter and Materials Physics, (88), 4.

http://dx.doi.org/10.1103/PhysRevB.88.045321

Copyright: American Physical Society

http://www.aps.org/

Postprint available at: Linköping University Electronic Press

http://urn.kb.se/resolve?urn=urn:nbn:se:liu:diva-92307 


\title{
Quantum dot asymmetry and the nature of excited hole states probed by the doubly positively charged exciton $X^{2+}$
}

\author{
Daniel Dufåker, ${ }^{1}$ K. F. Karlsson, ${ }^{1}$ L. O. Mereni, ${ }^{2}$ V. Dimastrodonato, ${ }^{2}$ G. Juska, ${ }^{2}$ E. Pelucchi, ${ }^{2}$ and P. O. Holtz ${ }^{1}$ \\ ${ }^{1}$ Linköping University, Department of Physics, Chemistry, and Biology (IFM), Semiconductor Materials, S-58183 Linköping, Sweden \\ ${ }^{2}$ Tyndall National Institute, University College Cork, Ireland
}

(Received 12 April 2013; published 29 July 2013)

\begin{abstract}
In this experimental and theoretical study, it was found that the emission pattern of the doubly positively charged exciton complex $X^{2+}$ strongly depends on the nature of the involved excited hole states as well as the quantum dot symmetry. The two-hole system in the final state of the $X^{2+}$ recombination for the investigated high-symmetry pyramidal InGaAs quantum dots does not exhibit the singlet-tripletlike arrangement previously observed for the two-electron counterpart. Instead, the final states exhibit two true doublets, in accordance with group-theoretical predictions. Asymmetry is manifested in the photoluminescence spectra of $X^{2+}$ by a significant splitting of one doublet, which is a spectral feature exhibited to some degree by all of the measured quantum dots. The analysis demonstrates that an external magnetic field elevates the symmetry of the quantum dots. This work highlights the exciton complex $X^{2+}$ as a very sensitive probe of the quantum dot shape as well as the nature of the involved quantum states. Thus, its spectral features are very suitable for an efficient uninvasive postgrowth symmetry characterization of quantum dots.
\end{abstract}

DOI: 10.1103/PhysRevB.88.045321

PACS number(s): 78.55.Cr, 73.21.La, 78.67.Hc

\section{INTRODUCTION}

Today nanometer-sized heterostructures are routinely fabricated by embedding a narrow band-gap semiconductor material into another material with a wider band gap. This creates a potential well for charge carriers, such as electrons $(e)$ and holes $(h)$, in the material at hand. If the charge carriers trapped in such a potential are restricted to move in all three dimensions, the structure is commonly named a quantum dot (QD). The optical emission spectra, as a result of recombining electrons and holes within the QD, are typically a discrete set of sharp peaks resembling atomic spectra. Quantum dots are thus often referred to as artificial atoms. ${ }^{1}$

In semiconductors, a single exciton, $X(1 e+1 h)$, is formed as a result of the Coulomb attraction within an electron-hole pair. Two bound excitons form a biexciton, $2 X(2 e+2 h)$. Highly charged excitons (excess of either electrons or holes) become possible only within QDs, due to the efficient confinement potential created for the charge carriers by the heterostructure. ${ }^{2,3}$ A wide range of negatively and positively charged exciton complexes have been spectrally identified. The recombination of the doubly negatively charged exciton $X^{2-}(3 e+1 h)$ leaves the quantum dot in the final states with two electrons accommodated in different energy levels. The resulting emission pattern with two well-resolved peaks is commonly understood as a consequence of the formation of singlet and triplet final states, analogous to the excited states of a helium atom. ${ }^{3}$ The peaks are typically split by $3-10 \mathrm{meV}$, corresponding to twice the electron-electron exchange energy $\left(\Delta_{e e}\right)$. The small electron-hole exchange interaction $\left(\Delta_{e h}\right)$ among the initial states results in a less significant fine structure of each peak, causing minor splittings that are about two orders of magnitude smaller than $\Delta_{e e}$. It was proposed that a two-hole system would follow the same singlet-triplet arrangement as the two-electron system, based on the analysis of an excited positive trion $X^{+}(1 e+2 h)$ with heavy holes. ${ }^{4}$ However, a more complete experimental and theoretical analysis of the doubly positively charged exciton $X^{2+}(1 e+3 h)$ for conventional Stranski-Krastanow (SK) grown QDs revealed that the final triplet states of the two-hole system are fundamentally different from the two-electron system, intrinsically split due to hole-hole exchange interactions $\left(\Delta_{h h}\right) .^{5,6}$

In this paper, we have investigated pyramidal QDs grown along the [111] direction in inverted tetrahedral recesses. Our investigation shows that for these QDs, the singlet-triplet model completely fails to describe the final two-hole system of a recombining $X^{2+}$ complex. Instead, we found that for a QD of point-group symmetry $C_{3 v}$, the two holes form two doublets, consistent with group-theoretical predictions and numerical computations. Furthermore, we demonstrate that approximate singlet-tripletlike arrangements may be formed, depending on the exact symmetry properties and degeneracy of the involved excited hole levels, in agreement with previous studies on SK QDs.

The advantage of the pyramidal QDs is their potential to possess the high $C_{3 v}$ symmetry, while the conventional SK QDs grown on a (001) plane are restricted to the lower $C_{2 v}$ symmetry. High-symmetry QDs are particularly interesting as emitters of polarization entangled photons. ${ }^{7,8}$ In this paper, we suggest that the $X^{2+}$ spectrum of pyramidal QDs is a sensitive tool to probe deviations from the ideal $C_{3 v}$ symmetry, with possible applications in postgrowth identification of QDs with sufficiently high symmetry.

\section{SAMPLE}

The $\mathrm{In}_{0.15} \mathrm{Ga}_{0.85} \mathrm{As} / \mathrm{AlGaAs}$ QD sample used for the experimental part of this study was grown on a prepatterned GaAs (111)B substrate by low-pressure metal organic vapor phase epitaxy (MOVPE). The 7.5 micron pitched pattern consists of inverted tetrahedral micropyramids. During growth, particular care is taken to monitor unintentional impurity levels. ${ }^{9-11}$ The QDs are self-formed at the bottom of the inverted pyramids during deposition of the $\operatorname{In}_{0.15} \mathrm{Ga}_{0.85}$ As layer due to capillarity effects and decomposition-rate anisotropy. ${ }^{12,13}$ Before and after growth of the QD layer, an $\mathrm{Al}_{30} \mathrm{Ga}_{70} \mathrm{As}$ barrier layer 


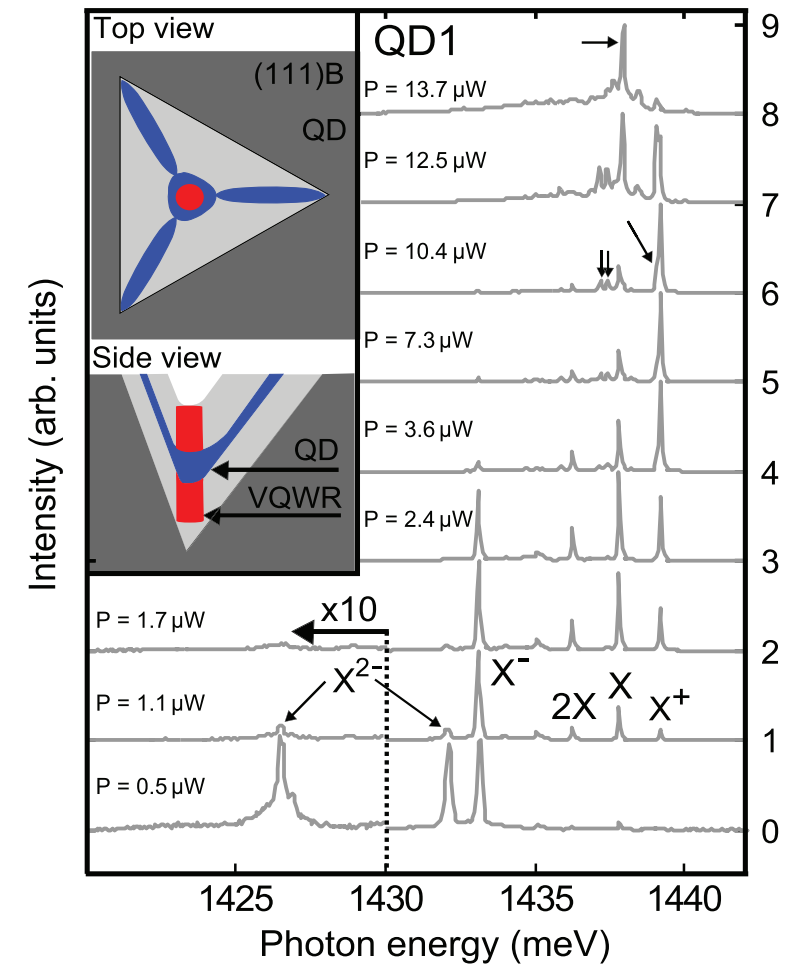

FIG. 1. (Color online) Excitation-power-dependent spectra measured for one QD, QD1. The spectra are normalized and shifted horizontally for clarity. Previously identified excitonic species are labeled according to conventions. Arrows in the top spectra indicate previously unidentified emission lines. The inset shows schematic top and side views of the pyramidal InGaAs/AlGaAs QD structure used in the study.

was grown (see the inset of Fig. 1). Due to alloy segregation in the barrier, the $\mathrm{Al}$ concentration in the vicinity of the QD is lowered and a vertical quantum wire (VQWR) is self-formed in the center of the pyramid. The sample was routinely back-etched after growth, a procedure in which the original GaAs substrate is removed to gain free upright standing pyramids, increasing the light-extraction efficiency from the QDs. ${ }^{14,15}$

\section{EXPERIMENTAL DETAILS}

The $\mathrm{In}_{0.15} \mathrm{Ga}_{0.85}$ As QDs were kept in a liquid helium cryostat at a temperature of $\sim 4 \mathrm{~K}$ in a microphotoluminescence $(\mu \mathrm{PL})$ setup and were excited individually with a Ti-sapphire laser of $732 \mathrm{~nm}$ wavelength. The laser was focused to a spot with a diameter of $\sim 2 \mu \mathrm{m}$ by means of a microscope objective with a numerical aperture of 0.55 . For a single grating monochromator (1200 grooves $/ \mathrm{mm}$ blazed for $750 \mathrm{~nm}$ and a focal length of $0.55 \mathrm{~m}$ ) equipped with a charge-coupled device (CCD) camera, the spectral resolution of $\sim 0.1 \mathrm{meV}$ was achieved. The average number of electrons and holes populating the QD could be controlled by the laser excitation power. The setup was equipped with a superconducting solenoid magnet allowing magnetic field measurements up to a field strength of $5 \mathrm{~T}$. Circular-polarization-resolved detection was enabled through the use of a rotatable Berek compensator, operating as a quarter-wave retardation plate, in conjunction with a fixed Glan-Thompson linear polarizer.

\section{IDENTIFICATION OF THE $X^{2+}$ AND $X^{3+}$ COMPLEXES}

Typical excitation-power $(P)$-dependent spectra for a pyramidal QD (QD1) are shown in Fig. 1. All peaks appearing at low-excitation power have been identified and labeled according to previous works on pyramidal QDs, where a gradual increase of the power shifted the charging of the QDs from $X^{5-}$ to $X^{+} .{ }^{2,16}$ The two expected peaks related to $X^{2-}$ are observed at the lowest excitation power in Fig. 1. For the highest excitation powers, four unidentified peaks appear (indicated by arrows in Fig. 1). These peaks are better exposed in Fig. 2, where an enlargement of four spectra of QD1 is shown together with the corresponding spectra for another QD (QD2). It should be noted that these peaks were observed for high-excitation powers for all ten QDs studied in this work. Three of the four unidentified peaks are correlated in intensity, exhibiting the same relative intensities as the power is changed, while the intensity of a fourth peak clearly has a different power dependence than the others. Since a gentle increase of the excitation power previously has resulted in a successive change of the charging state in these types of QDs, from $X^{5-}$ to $X^{+}$, it is reasonable to ascribe the correlated set of three peaks to the doubly charged exciton, $X^{2+}$, while the single peak, appearing at even higher excitation power, is ascribed to the triply charged exciton, $X^{3+}$. This identification will be confirmed by a comprehensive analysis performed in the following sections.

It should be emphasized that the $X^{2+}$ complex previously has been experimentally and theoretically studied for conventional SK QDs. ${ }^{5}$ The reported $\mu \mathrm{PL}$ spectrum of $X^{2+}$

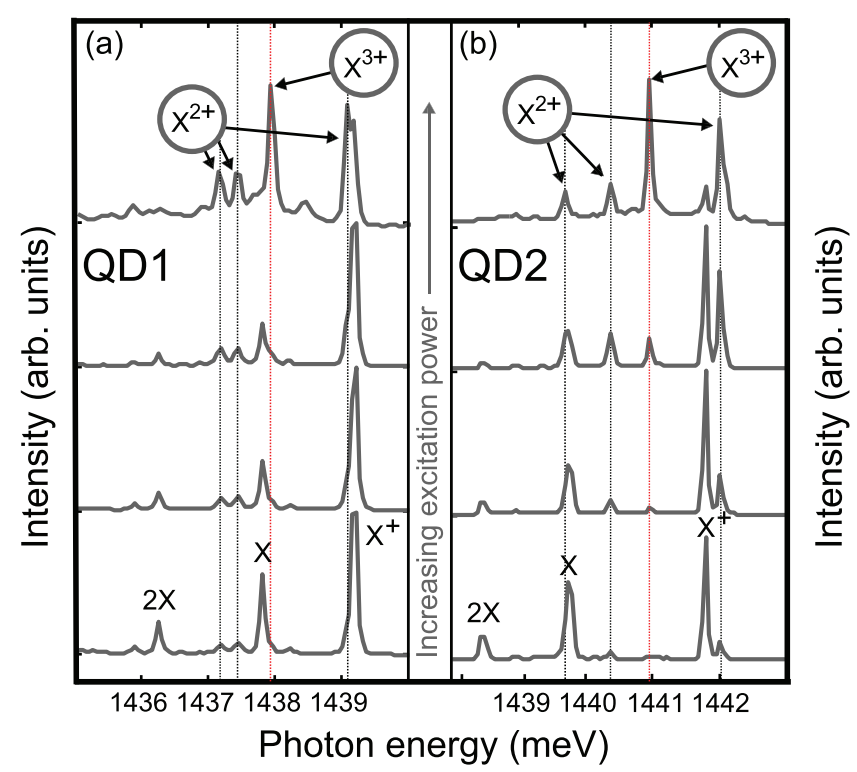

FIG. 2. (Color online) Excitation-power dependence measured for (a) QD1 and (b) QD2. The spectra are normalized and shifted horizontally for clarity. The weak dotted lines serve as a guide to the eye and mark the positions of the three components in the optical decay of the doubly charged exciton, $X^{2+}$, and the single component in the optical decay of the triply charged exciton, $X^{3+}$. 
reveals a singlet-tripletlike level arrangement of the two remaining charge carriers in the final states of the recombination. This is in analogy with $X^{2-}$, but, in contrast to $X^{2-}$, its positive counterpart $X^{2+}$ exhibits one well-resolved splitting of the tripletlike states. This splitting was explained to be a consequence of the fact that the holes follow the addition rule of spin- $3 / 2$ particles, which is different from the addition rule of spin- $1 / 2$ electrons, which results in true singlet-triplet states. ${ }^{5}$ Hence, $X^{2+}$ exhibits, according to their explanation, three spectral peaks, while $X^{2-}$ exhibits two peaks (the small fine-structure splitting of the initial state created by the electron-hole exchange interaction is here neglected).

In this work, the $\mu \mathrm{PL}$ spectrum of $X^{2+}$ of the pyramidal QDs exhibits three components (see Fig. 2), but the spectral pattern is strikingly different from the previously reported one for SK QDs. Instead of two high-energy components, originating from the tripletlike states, and one low-energy component, originating from the singletlike states, the investigated pyramidal QDs exhibit one high-energy component and two, only slightly separated, low-energy components. The measured difference in energy [as averaged from 10 QDs ( \pm 1 standard deviation)] between the single high-energy component and the average of the two low-energy components is $1.9( \pm 0.3) \mathrm{meV}$ and the splitting between the two low-energy components is $0.8( \pm 0.7) \mathrm{meV}$. Thus, the energy pattern is reversed compared to the SK dots, while the PL intensities are similar in both cases, with the strongest intensity for the component with the highest energy.

\section{GROUP-THEORETICAL CONSIDERATIONS AND COMPUTATIONS}

We start our analysis of the doubly charged exciton $X^{2+}$ with a group-theoretical approach based on an ideal QD belonging to point-group symmetry $C_{3 v}$ and neglecting Coulomb mixing between the hole levels. Each quantum eigenstate can be labeled by a corresponding irreducible representation of the point group, following the notations of Ref. 17. For a $C_{3 v} \mathrm{QD}$, the label of the electron ground level is known to be $E_{1 / 2}$. The holes of $X^{2+}$ must be distributed among two hole levels, and each level can in $C_{3 v}$ only be labeled with either $E_{1 / 2}$ or $E_{3 / 2}$. Previous studies of similar but shallower pyramidal InGaAs QDs concluded that the heavy-holelike ground level should be labeled $E_{3 / 2}{ }^{7,18} \mathrm{We}$ assume that the single electron and two of the holes are accommodated in their respective ground levels, i.e., $e_{1}$ and $h_{1}$, while the third hole is in an arbitrary excited hole level $h_{n}(n>1)$. Under these conditions, only two sorts of $X^{2+}$ complexes can be formed, with each hole level, $h_{n}$, labeled either $E_{1 / 2}$ or $E_{3 / 2}$. The initial and final product states of $X^{2+}$ with the resulting possible PL transitions for these two cases were derived according to Ref. 18 and presented in Fig. 3(a). Already at this stage, the group-theoretical approach manifested in the decay schemes reveals some interesting features: The final states of $X^{2+}$ are expected to be arranged either as two doublets (for $h_{n}: E_{1 / 2}$ ) or as four singlets (for $h_{n}: E_{3 / 2}$ ). Hence, a true singlet-triplet arrangement cannot, in general, be formed for a $C_{3 v}$-symmetry QD. It was concluded in a previous study that the elevated symmetry $D_{3 h}$, to a very good approximation, describes the excitonic complexes in pyramidal QDs. ${ }^{7}$ For completeness,

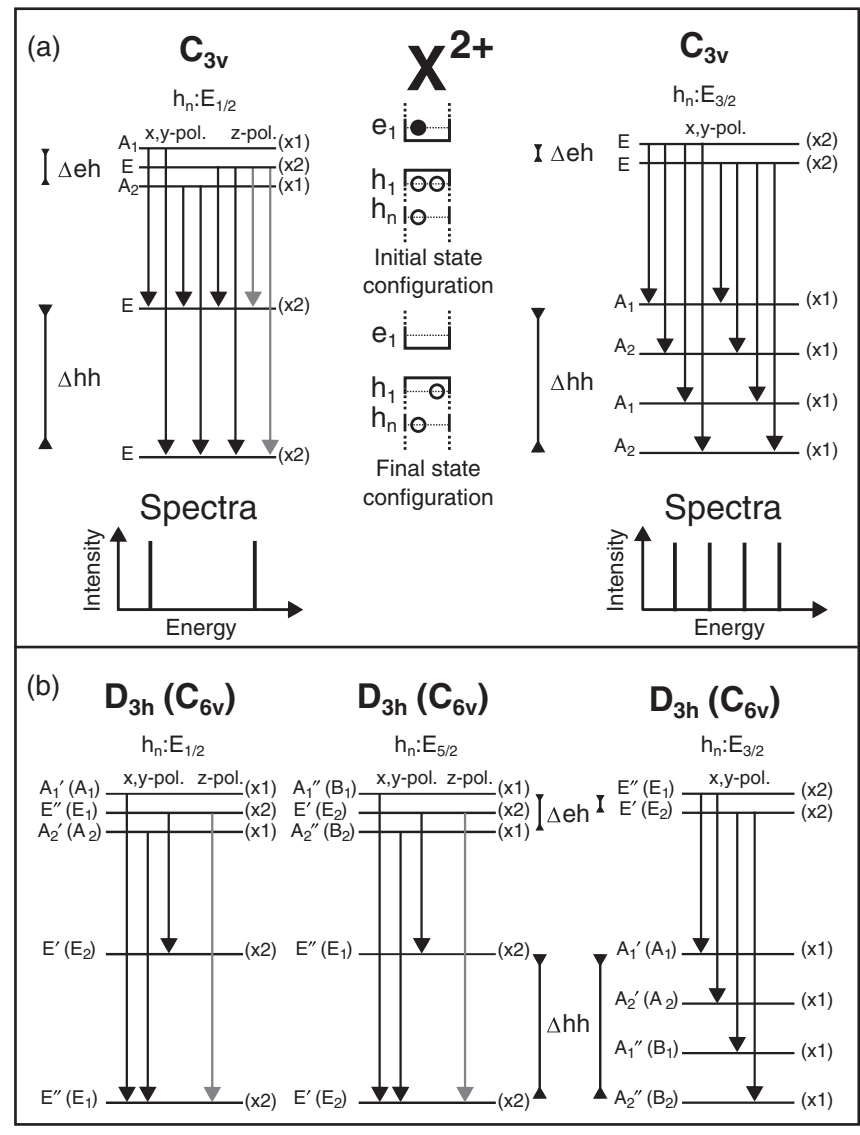

FIG. 3. Derived decay schemes for the doubly positively charged exciton $X^{2+}$, for the different point-group symmetries $C_{3 v}$ and the isomorphic $D_{3 h}$ and $C_{6 v}$. The decay scheme is based on different symmetries of the excited hole wave function $h_{n}$, according to the irreducible representations $E_{1 / 2}$ or $E_{3 / 2}$ of $C_{3 v}$, as well as $E_{1 / 2}, E_{5 / 2}$, or $E_{3 / 2}$ of $D_{3 h}$ and $C_{6 v}$. The electron and hole ground levels, $e_{1}$ and $h_{1}$, have in all cases been labeled $E_{1 / 2}$ and $E_{3 / 2}$, respectively. The $x$ - and $y$-polarized emissions are labeled $E, E^{\prime \prime}$, and $E_{1}$ in $C_{3 v}, D_{3 h}$, and $C_{6 v}$, respectively. The insets in the middle of (a) show the corresponding single-particle configuration for the initial and final states of $X^{2+}$. The bottom portion of (a) shows one possible spectrum that is compatible with the corresponding decay scheme above, where the electron-hole exchange splitting of the initial states is neglected.

also the corresponding decay diagrams for $D_{3 h}$ as well as the isomorphic point group $C_{6 v}$ are shown in Fig. 3(b). $D_{3 h}$ and $C_{6 v}$ allow a third type of hole level, labeled $E_{5 / 2}$. However, as seen in Fig. 3(b), the decay scheme of $X^{2+}$ is identical, irrespective of whether $h_{n}$ bears the label $E_{1 / 2}$ or $E_{5 / 2}$. Moreover, if the small fine-structure splitting $\Delta_{e h}$ of the initial states is neglected, then the spectra predicted from the elevated symmetries $D_{3 h}$ and $C_{6 v}$ are identical with the spectra predicted from $C_{3 v}$ [see Fig. 3(a)].

In order to compute the involved hole states and Coulomb related energy splittings, an $8 \times 8$ band $\mathbf{k} \cdot \mathbf{p}$ approach was used. The $\mathrm{In}_{0.15} \mathrm{Ga}_{0.85}$ As QD model is adapted from Ref. 19, where the same QDs were modeled as a cylinder, $6 \mathrm{~nm}$ high and $24 \mathrm{~nm}$ in diameter, intersected by an AlGaAs VQWR along the [111] direction for studies of the exciton-phonon interaction of $X^{-}, X$, and $X^{+}$. The In concentration in the QD is assumed to gradually decrease, proportionally to minus the 


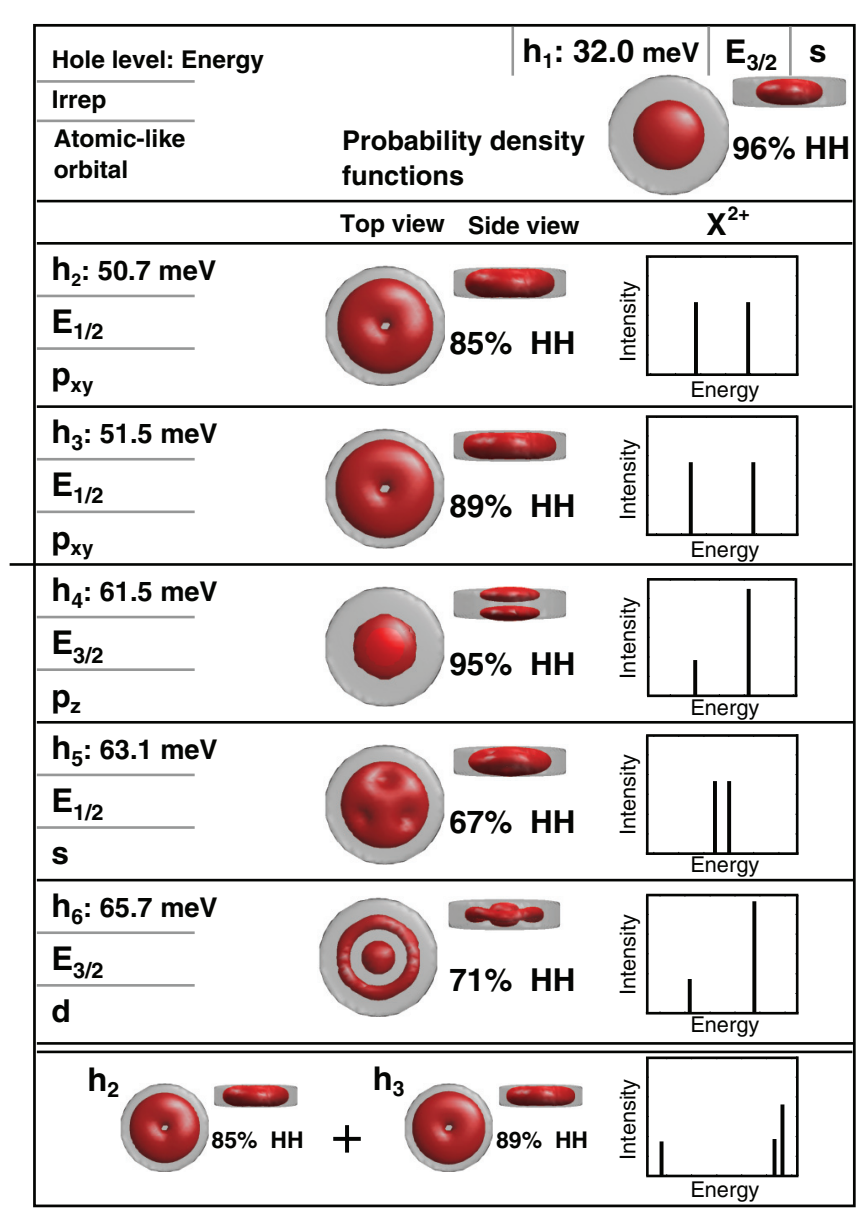

FIG. 4. (Color online) Calculated probability density functions for the ground hole level $h_{1}$ (top panel) and for the excited hole levels $h_{2}$ to $h_{6}$ (subsequent panels) of a cylindrical QD with the symmetry axis along [111] (shown for both top and side view). The valence-band energy (with respect to the valence-band edge of GaAs), the atomiclike orbital of the envelope function, the heavy-hole content with respect to [111], and the labels of $C_{3 v}$ irreducible representations for each hole level are also indicated in the panels. Idealized spectra of the optical transitions computed for $X^{2+}$ with two $h_{1}$ holes and the third hole, $h_{n}$, in a single excited hole state $(n>1)$ are shown to the right. A spectrum was also computed by including both $h_{2}$ and $h_{3}$ as possible levels for the third hole (bottom panel). The $x$ axis spans over $12 \mathrm{meV}$ in each spectrum for easy comparison. The small electronhole exchange interaction was neglected in the computations.

square of the radius, from $20 \%$ at the center down to $10 \%$ at the circumference. The VQWR and surrounding bulk have the Al concentration set to $5 \%$ and $25 \%$, respectively. The strain in the QDs stemming from the lattice mismatch is accounted for by continuum elastic theory. The piezoelectric field was not included in the calculations, since its exact value is somewhat ambiguous. ${ }^{20}$ The shape of the QD model is of high symmetry, $D_{\infty h}$, but the confined QD states are strictly limited to $C_{3 v}$ due to the intersection with the $T_{d}$ symmetry of the bulk $\mathbf{k} \cdot \mathbf{p}$ Hamiltonian. The single-particle hole levels, $h_{1}$ to $h_{6}$, achieved from the calculations are presented in Fig. 4. All hole states are predominantly of heavy-hole $(\mathrm{HH})$ character with respect to [111]. The hole ground states of the QD have $s$-like envelope wave functions, confirmed to have the label $E_{3 / 2}$. The two first excited hole levels $h_{2}$ and $h_{3}$ are quasi-degenerate (separated by $<1 \mathrm{meV}$ ) with lateral $p$-like envelope wave functions $\left(p_{x y}\right)$ and are labeled $E_{1 / 2}$. The next level, $h_{4}$, corresponds to vertical $p$-like envelope wave functions $\left(p_{z}\right)$ with label $E_{3 / 2}$. The fifth hole level, $h_{5}$, is an analog to the $s$-like light hole ground level with the label $E_{1 / 2}$. Although $h_{5}$ is primarily of $\mathrm{HH}$ character, it corresponds to the hole states with the smallest amount of $\mathrm{HH}$ character. The final hole level investigated has $d$-like envelope wave functions and is labeled $E_{3 / 2}$. Plausible candidates for the excited hole level $h_{n}$ involved in $X^{2+}$ are the $p$-like $h_{2}$ and $h_{3}$ levels, known to be the first excited levels in flat SK QDs, ${ }^{5}$ or the $s$-like $h_{5}$ level, which was found to be the first excited level in shallow pyramidal QDs. ${ }^{21}$ For thicker QDs, the $h_{4}\left(p_{z}\right)$ level is expected to shift in energy and eventually become the first excited level.

The electron-hole and hole-hole direct Coulomb interaction matrix elements, as well as the long-range hole-hole Coulomb exchange scattering matrix elements, are computed from the single-particle states and subsequently injected into a manybody configuration interaction (CI) Hamiltonian. ${ }^{22}$ In the quest to distinguish between the two possible hole labels and to determine the actual hole level involved in the recombination, we have computed idealized spectra of $X^{2+}$ with the electron in $e_{1}$, two holes in $h_{1}$, and the third hole in an isolated excited level of $h_{n}$. The spectra obtained by neglecting the small electron-hole exchange interaction $(\Delta e h)$ are shown on the right-hand side in Fig. 4. Since $h_{2}$ and $h_{3}$ are close in energy, the states of $h_{2}$ and $h_{3}$ are expected to be strongly mixed and it may not be appropriate to consider them as isolated. Therefore, the lowest panel of Fig. 4 shows the spectrum of a more realistic case, where the third hole is in a mixed state of $h_{2}$ and $h_{3}$. The computed spectra in Fig. 4 with the third isolated hole in $h_{2}, h_{3}$, or $h_{5}$, labeled $E_{1 / 2}$, all exhibit two peaks, since the final states of the $X^{2+}$ recombination in this case consist of two doublets according to the group-theoretical predictions presented in Fig. 3(a). On the other hand, for the isolated excited hole levels of $h_{4}$ and $h_{6}$, labeled $E_{3 / 2}$, it is clear that only two transitions are observed in the computed spectra instead of the predicted four in Fig. 3(a). The reason for this is that the final states of the recombination of $X^{2+}$ in this case have an approximate singlet-tripletlike arrangement, where the splittings of the states in the quasitriplet is about $1-10 \mu \mathrm{eV}$ and remains unresolved in the spectra of Fig. 4. Furthermore, the interactions between $h_{2}$ and $h_{3}$ also yield a singlet-tripletlike arrangement, but in this case the splitting of the quasitriplet is significantly larger $(\sim 600 \mu \mathrm{eV})$ and clearly resolved in the computed spectrum shown in the bottom panel of Fig. 4. It is obvious that none of these computed spectra, in a satisfactory way, display the features of the experimental data in Fig. 2. Only the spectrum in the bottom panel of Fig. 4 exhibits the same number of peaks as the measured $\mu \mathrm{PL}$ spectra, but the computed energy pattern of the peaks is reversed compared to the experiments, with two peaks, only slightly split, on the high-energy side instead of the low-energy side. All other computed spectra with an isolated excited hole state exhibit only two emission lines, originating either from two true doublets or from approximate singlet-tripletlike final states.

The group-theoretical considerations as well as the numerical computations have so far been based on an ideally 
symmetric QD (i.e., $C_{3 v}$ ), but the actual measurements were performed on real QDs, which are unlikely to be perfectly symmetric. $^{18}$ Any anisotropic perturbation that lowers the symmetry from $C_{3 v}$ splits the degenerate $E$ states (see Fig. 3), and it is the splittings of the final states that are most significant in a PL spectrum, since the asymmetry-induced splitting of the initial states due to electron-hole exchange interactions typically is very small $(<100 \mu \mathrm{eV})$ and difficult to spectrally resolve. It should be noted that symmetry breaking for the case of mixed excited states (see the bottom panel of Fig. 4) yields more spectral components than the three experimentally observed. For the computed singlet-tripletlike states with the excited hole in an $E_{3 / 2}$ level (see the panels of $h_{4}$ and $h_{6}$ in Fig. 4), the spectral component of lowest energy is originating from the singlet, which cannot split further by asymmetry. This suggests that the two slightly split low-energy components observed in the $X^{2+} \mu \mathrm{PL}$ spectrum originate from a doublet, slightly split by asymmetry. This would imply that a relatively isolated excited hole level with label $E_{1 / 2}$ in the symmetric limit is involved in the formation of $X^{2+}$ (see the panels of $h_{2}, h_{3}$, and $h_{5}$ in Fig. 4). Then, in principle, a splitting of the upper spectral component of $X^{2+}$ is also expected, since it also originates from a doublet, which splits upon symmetry breaking. Such a symmetry breaking was investigated in detail in Ref. 7 for the initial states of an excited positive trion, which, like the final states of $X^{2+}$, also involves one hole in a ground state labeled $E_{3 / 2}$ and an excited hole in a level labeled $E_{1 / 2}$. It was found for this two-hole system that only the high-energy doublet splits appreciably upon weak symmetry breaking, whereas the splitting of the low-energy doublet was less than $2 \mu \mathrm{eV}$. A similar behavior for the final state of $X^{2+}$ implies that only the low-energy spectral peak splits sufficiently to be spectrally resolved by asymmetry, consistent with the experiments.

\section{THE NATURE OF THE EXCITED HOLE LEVEL}

It follows from the discussion above that the excited hole states involved in $X^{2+}$ belong to a relatively isolated hole level $h_{n}$ with label $E_{1 / 2}$. Most probable is that the observed emission of $X^{2+}$ originates from its ground state, in which both hole states of $h_{1}$ and one hole state of $h_{2}$ are populated. According to the current QD model, $h_{2}$ is not isolated, but is quasi-degenerate with $h_{3}$. In order to clarify whether or not $h_{2}$ is isolated for the measured QDs, the weak luminescence observed energetically above the main emission was investigated experimentally. Two $\mu \mathrm{PL}$ spectra measured for different excitation powers are shown in Fig. 5. The low power spectrum $(P=0.7 \mu \mathrm{W})$ is dominated by the negatively charged exciton $X^{-}$and the neutral exciton $X$, and two corresponding peaks, about 100 times weaker, appear $\sim 15 \mathrm{meV}$ above the main emission. The peaks at higher energy are interpreted as the luminescence from the excited complexes $X^{-}$and $X$, with the hole in an excited level $h_{m}$ (see the insets of Fig. 5). The intensities of these transitions are weak, since the probability of finding the hole in an excited state is very small at the low temperature of the experiments. At higher excitation powers, the formation of $X^{2+}$ and $X^{3+}$ implies state filling of the holes up to $h_{2}$, and relaxation of holes from $h_{2}$ to $h_{1}$ is then prohibited due

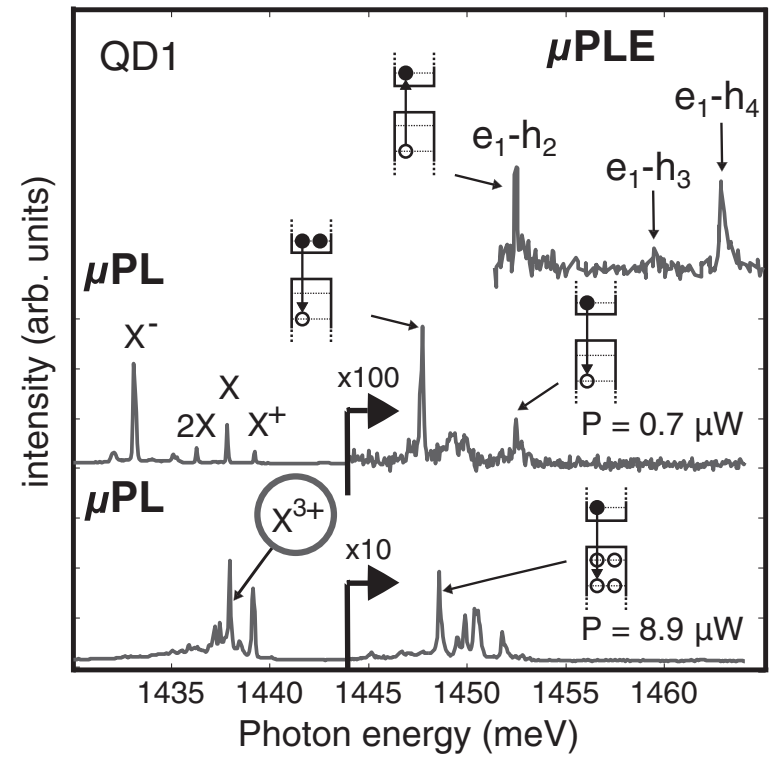

FIG. 5. Measured micro-photoluminescence excitation spectrum ( $\mu$ PLE) with detection on the neutral exciton $X$ is shown together with measured microphotoluminescence ( $\mu \mathrm{PL})$ spectra for two different excitation powers. Luminescence from exciton complexes with the recombining hole in an excited state are observed in the energy range $1445-1455 \mathrm{meV}$ (enhanced by a factor of 10 or 100). The insets next to the some $\mu \mathrm{PL}$ and $\mu \mathrm{PLE}$ peaks schematically illustrate the involved transition.

to the Pauli exclusion principle. This implies that $h_{2}$ becomes efficiently populated with holes despite the low temperature, and the relative intensity of the $h_{2}$-related PL emission should, consequently, increase significantly with the appearance of $X^{2+}$ and $X^{3+}$ in the main emission. Indeed, the high power spectrum $(P=8.9 \mu \mathrm{W})$ of Fig. 5 shows an enhancement of the relative intensity by about one order of magnitude with the excitation of $X^{2+}$ and $X^{3+}$, confirming that the emission observed $\sim 15 \mathrm{meV}$ above the main emission is related to the first excited hole level $h_{2}$. The fact that the high-energy emission of the QD with state filling is 10 times weaker than the main emission simply reflects that the probability for $e_{1}-h_{2}$ transitions is smaller than for $e_{1}-h_{1}$ transitions.

Additional excited states, positioned at higher energy, are probed by micro-photoluminescence excitation ( $\mu$ PLE) spectroscopy of $X$. In addition to the first excited exciton state $e_{1}-$ $h_{2}$, at exactly the same energy as the corresponding PL peak, the $\mu$ PLE spectrum in Fig. 5 reveals resonances located 7 and $10 \mathrm{meV}$ above the first excited state, attributed to the transitions $e_{1}-h_{3}$ and $e_{1}-h_{4}$, respectively. Hence, the previously discussed indications of the fact that it is a relatively isolated excited hole level, which is involved in $X^{2+}$, are supported by these experimental results.

\section{IMPROVED MODEL}

The current model, where the QD is described as a simple cylinder, yields quasi-degenerate excited hole levels $h_{2}$ and $h_{3}$, inconsistent with the presented experimental results, that indicate an isolated $h_{2}$ level. Moreover, the current model 

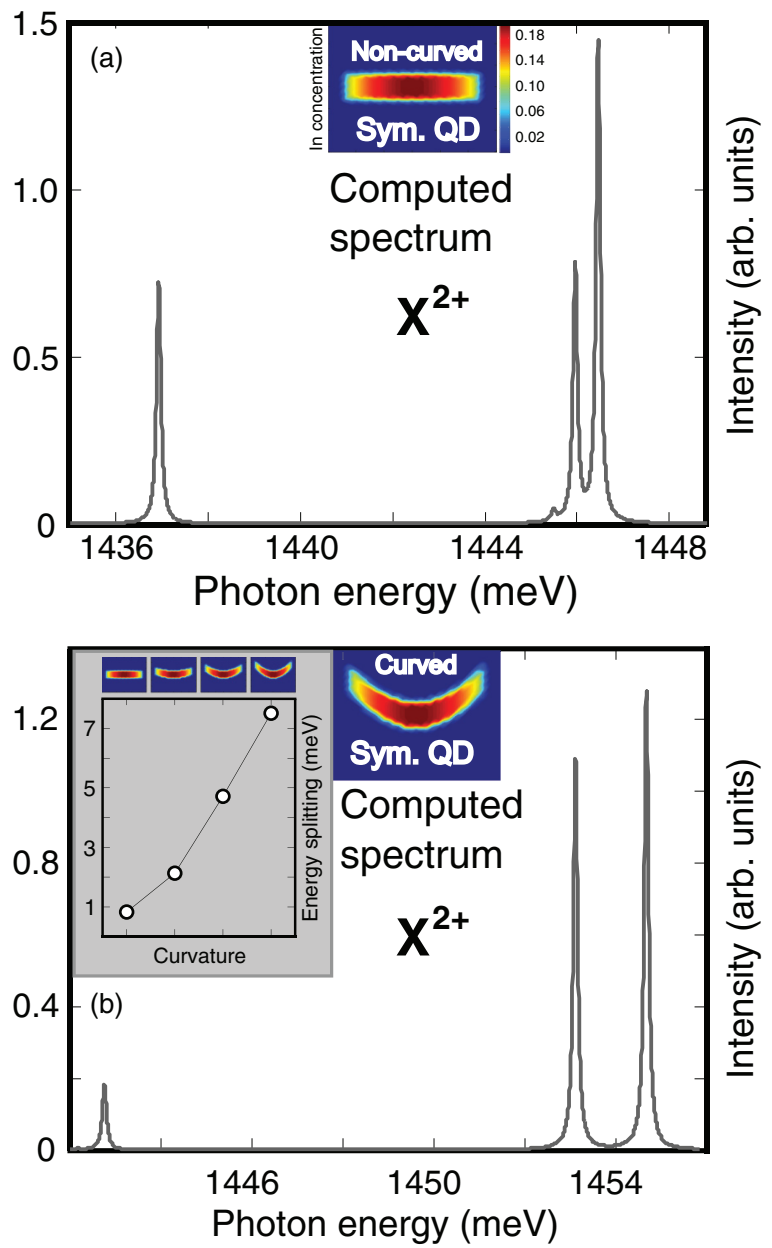

FIG. 6. (Color online) Computed optical spectra of $X^{2+}$ for a symmetric QD (a) without and (b) with a parabolic curvature. The inset in (a) shows a side view of the QD curvature with the In concentration used for the computations. The inset of (b) shows the energy splitting between the hole levels $h_{2}$ and $h_{3}$ as a function of the parabolic curvature, along with a side view of the QD curvature for the particular QD.

predicts vanishing optical transition probability for $e_{1}-h_{2}$, while the $\mu \mathrm{PL}$ spectra clearly demonstrate an intensity up to about $10 \%$ of the main emission. The cylindrical model of the pyramidal QDs is obviously oversimplified and does not correctly reflect the features of the excited hole states. Real pyramidal QDs do exhibit a curvature created by a sharp selflimiting profile in the AlGaAs bottom of the inverted pyramid during growth. ${ }^{23}$ By introducing a parabolic curvature on the cylinder, creating a bowl-shaped QD model, the computed energy separation between the two $p$-like hole levels $h_{2}$ and $h_{3}$ increases, and the optical transition probability for both $e_{1}-h_{2}$ and $e_{1}-h_{3}$ becomes enhanced. In the inset of Fig. 6(b), the energy separation between $h_{2}$ and $h_{3}$ is plotted as a function of the QD curvature. The energy separation increases from $\sim 0.8 \mathrm{meV}$ for the flat QD up to $\sim 7.5 \mathrm{meV}$ for the strongest curvature computed simultaneously as the intensity of the $x, y$-polarized transitions $e_{1}-h_{2}$ is enhanced from zero up to $\sim 8 \%$ of the main transition $e_{1}-h_{1}$.

Full CI computations of $X^{2+}$, including one electron level $e_{1}$ and all six hole levels $h_{1}-h_{6}$ (i.e., in total, 12 hole states

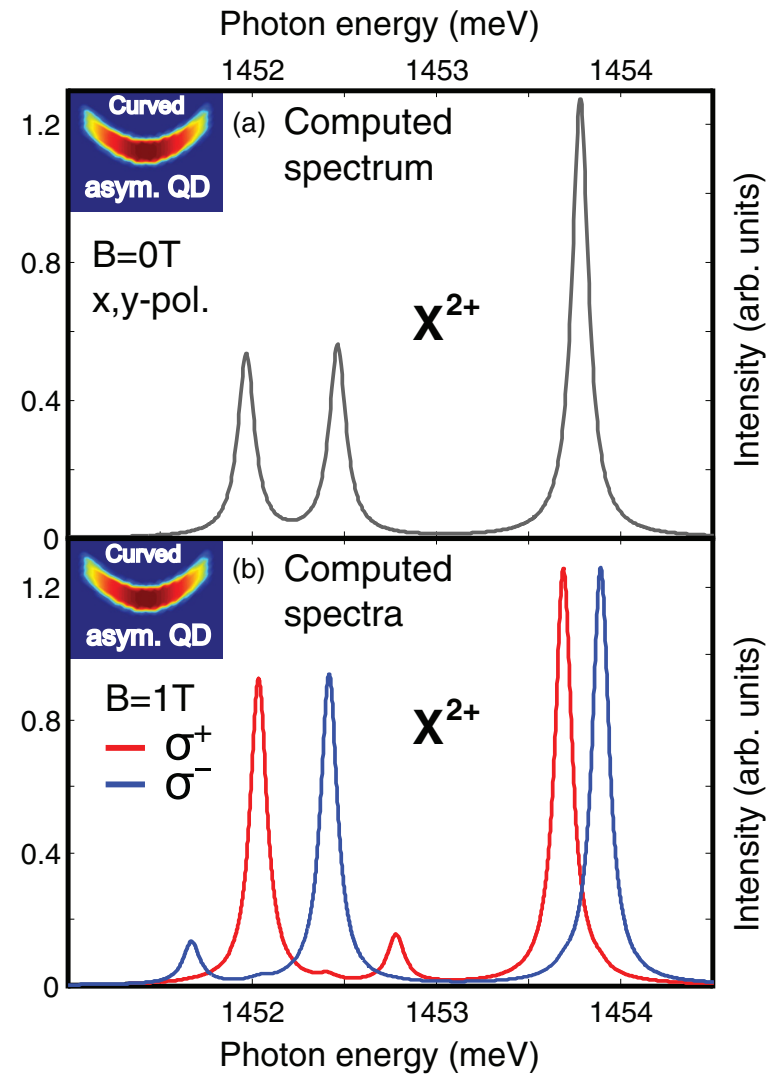

FIG. 7. (Color online) Computed optical spectra of $X^{2+}$ in a curved and slightly asymmetric QD (a) without and (b) with a magnetic field of strength $B=1 \mathrm{~T}$. The magnetic field spectra are presented for the two circular polarizations $\sigma^{+}$and $\sigma^{-}$.

due to Kramers degeneracy), for both the flat and curved QD model, give the optical transition spectra shown in Fig. 6 (where the electron-hole exchange interaction is neglected). Not surprisingly, the spectrum of the flat QD in Fig. 6(b) is dominated by the singlet-tripletlike arrangement in the final state, due to the quasi-degenerate hole levels $h_{2}$ and $h_{3}$. As a curvature is introduced and $h_{2}$ becomes more isolated, the low-energy peak is redshifted away from the main peaks, with fading intensity, while the two main peaks approach equal intensities, as previously predicted for a $C_{3 v} \mathrm{QD}$ and an isolated excited $E_{1 / 2}$ hole level with two true doublets in the final state. In this case, the weak low-energy peak corresponds to the small probability to find the third hole of $X^{2+}$ in $h_{3}$ immediately after recombination.

A weak symmetry breaking of the numerical QD model was accomplished by modifying the circular lateral shape of the dot to become slightly elliptic, with the lengths of the main and minor axes at 25.25 and $24 \mathrm{~nm}$, respectively; the computed main optical transitions of $X^{2+}$ of such an asymmetric QD are shown in Fig. 7(a). It is clear from the figure that asymmetry induces a splitting of the low-energy peak into two separate components. The computed spectrum, with one high-energy peak with dominating intensity and two weaker peaks located $\sim 2 \mathrm{meV}$ below, corresponds very well with the experimental data presented above [see Figs. 2 and 7(a)]. Note that the splitting of the low-energy peak originates from a splitting 


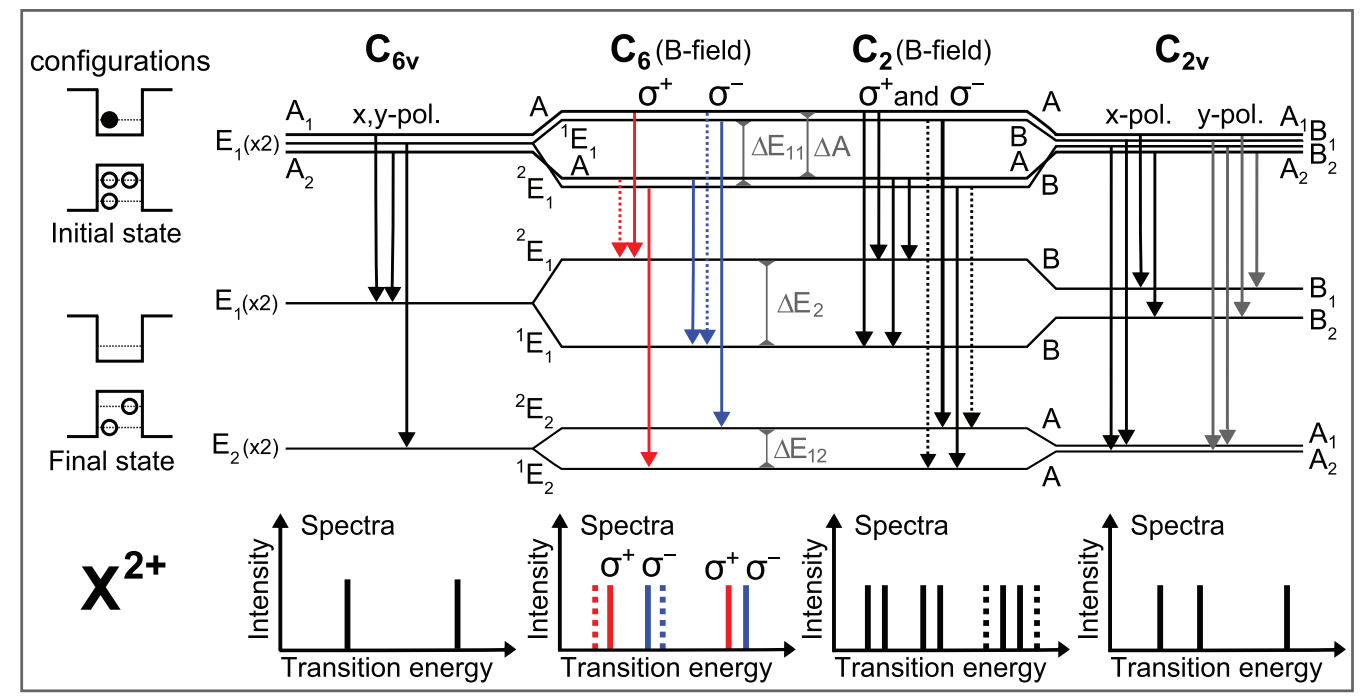

FIG. 8. (Color online) The dominant initial- and final-state single-particle configurations of the doubly positively charged exciton $X^{2+}$ are shown together with product states and the polarized radiative decay paths. In addition, the corresponding predicted optical recombination spectra connected to each of the symmetries $C_{6 v}, C_{6}, C_{2}$, and $C_{2 v}$, respectively, are shown underneath each decay scheme. The $z$-polarized transitions are not included. The labels of the $x$ - and $y$-polarized and the circularly polarized emission $\sigma^{+}$and $\sigma^{-}$are $E_{1}: x, y$ in $C_{6 v}, B_{1}: x$ and $B_{2}: y$ in $C_{2 v}, \sigma^{+}:{ }^{2} E_{1}$ and $\sigma^{-}:{ }^{1} E_{1}$ in $C_{6 v}$, and $\sigma^{+}, \sigma^{-}$: both $B$ in $C_{2}$. The dotted lines in the decay scheme correspond to peaks for which the computed intensity is significantly lower compared to the other peaks. The decay schemes are created in consistency with the computed as well as the experimental results. The exact order of the two initial product states $A_{1}$ and $A_{2}$ is not determined. The bottom panel shows one possible spectrum that is compatible with the corresponding decay scheme above, where the electron-hole exchange splitting of the initial states is neglected.

of the excited state of the two-hole system in the final state. Like previously reported for shallower pyramidal QDs, ${ }^{7}$ the asymmetry-induced splitting of the ground two-hole state is extremely small $(0.6 \mu \mathrm{eV})$ also here. One very important conclusion that can be made is that the energy separation between the two low-energy peaks of $X^{2+}$ is an efficient probe of the actual QD asymmetry, and this exciton complex can conveniently be used to distinguish asymmetric QDs from the symmetric ones. ${ }^{24}$ It should be noted that the curved QD model is incompatible with the proposed symmetry elevation towards $D_{3 h}$, which incorporates an approximate horizontal symmetry plane. ${ }^{7,18}$ However, symmetry elevation towards $C_{6 v}$ remains compatible with the curved model, and the excitonic decay schemes for $D_{3 h}$ and $C_{6 v}$ are identical due to isomorphy between the two point groups.

\section{MAGNETIC FIELD DEPENDENCE}

Applying an external magnetic field lowers the symmetry of the QD states, which may give rise to splittings and additional peaks in the recombination spectrum. A $C_{3 v}$-symmetry QD becomes $C_{3}$ for a magnetic field oriented along the dot symmetry axis, and all the degenerate $E$ states will split (Zeeman splitting). Correspondingly, the elevated symmetries $D_{3 h}$ and $C_{6 v}$ are reduced to $C_{3 h}$ and $C_{6}$, respectively. It is interesting to note that the point groups of the two elevated symmetries remain isomorphic also in the presence of a magnetic field, i.e., $C_{3 h}$ is isomorphic with $C_{6}$. Since the model for a symmetric QD is compatible with the elevation towards $C_{6 v}$, this will be the starting point of our group-theoretical analysis. The elevated symmetry $C_{6 v}$ allows for subduction to $C_{2 v}$ for the analysis of asymmetric QDs. It has previously been demonstrated that weakly asymmetric pyramidal QDs can be understood as approximately $C_{2 v}$, although the actual symmetry is $C_{s}$ or lower. $^{7}$

The derived decay diagram of $X^{2+}$ for $C_{6 v}$ for $e_{1}, h_{1}$, and $h_{2}$ labeled $E_{1 / 2}, E_{3 / 2}$, and $E_{1 / 2}$, respectively, is shown again in Fig. 8, together with relevant diagrams of lower symmetries obtained by subduction. The energy ordering of the involved states has been chosen to be consistent with the numerical results and the experimental data to be presented below. It should be stressed that the $z$-polarized transitions have been excluded from the decay schemes in Fig. 8, since the intensities of these lines are expected to be very weak due to the strong heavy-hole character of the recombining hole in $h_{1}$. Moreover, the $z$ polarization is not probed in our experiments.

If the small fine-structure splittings of the initial states due to electron-hole exchange interactions are neglected, then the idealized $X^{2+}$ recombination spectrum for a symmetric QD with elevated symmetry $C_{6 v}$ consists of two well-resolved peaks. For an asymmetric QD, it was previously discussed and demonstrated that the energy splitting of the two lowest-lying final states is extremely small, which yields three well-resolved spectral components, as seen in the computed spectra of $X^{2+}$ shown in Fig. 7(a) as well as in the measured $\mu \mathrm{PL}$ spectra shown in Fig. 2. This means that only the final states $B_{1}$ and $B_{2}$ split appreciably, while $A_{1}$ and $A_{2}$ remain quasi-degenerate under $C_{2 v}$ (see Fig. 8).

When the magnetic field is applied, the main effects of the symmetry transition from $C_{6 v}$ to $C_{6}$ are the splittings of the degenerate $E_{1}$ and $E_{2}$ states, for both the initial and the 
final states. Spectrally, this implies that the high-energy peak splits into two components, while the low-energy peak splits into four components, with all components circularly polarized either $\sigma^{+}$or $\sigma^{-}$. On the other hand, the predicted spectrum for $C_{3 v}$ with a magnetic-field-induced transition to $C_{3}$ consists of six components in each polarization $\sigma^{+}$or $\sigma^{-}$(not shown). For an asymmetric $C_{2 v}$ QD, there are no strict degenerate states, and the magnetic field will merely shift the states in consistency with subduction from $C_{6}$ to $C_{2}$. Spectrally, this results in a well-resolved splitting of the low-energy peaks of $X^{2+}$, while the high-energy peak splits into four components. Unlike the symmetric case, all components are active in both $\sigma^{+}$and $\sigma^{-}$polarization for $C_{2}$ symmetry.

Magnetic field effects were included in the $\mathbf{k} \cdot \mathbf{p}$ Hamiltonian by a gauge-invariant vector potential according to Ref. 25 and the Landé $g$ factors according to Ref. 26. The computed spectra of $X^{2+}$ for the weakly asymmetric QD in an external magnetic field ( $B=1 \mathrm{~T})$ applied along [111] is shown in Fig. 7(b), exhibiting six emission peaks with a high degree of circular polarization: three $\sigma^{+}$and three $\sigma^{-}$. The decay scheme in Fig. 8 for $C_{2}$ actually predicts eight peaks in total, but the intensity of two transitions (black dotted lines) are, according to the computations, about three orders of magnitude weaker than the other six, and are therefore not visible in the computed spectrum. Note that the remaining six computed peaks are not strictly $\sigma^{+}$or $\sigma^{-}$, but a weak mixture of both, consistent with the group-theoretical prediction in Fig. 8. Two of the six optically active components are significantly weaker than the other four, and they become further suppressed and eventually vanish as the field increases, while the degree of circular polarization of the remaining four lines approaches unity (at $2 \mathrm{~T}$, the intensity of the weak components is only $\sim 5 \%$ of the intensity of the four strong components). Thereby the spectrum of the asymmetric QD is approaching the corresponding spectrum of a symmetric QD at elevated field strengths, as the computed intensities of two of the transitions predicted for $C_{6}$ (dotted lines in Fig. 8) are very weak. In fact, the $C_{6}$ transitions represented by the dotted lines in Fig. 8 exhibit zero intensity for vanishing $V_{e h}$, but for realistic values of $V_{e h}$, the corresponding computed intensity is only about $2 \%$ of the main transition intensity at $B=1 \mathrm{~T}$, with a rapid decrease for further increased field strengths. Thus, there are only four components expected to be experimentally resolved at high magnetic field strengths.

The $\mu$ PL spectra of $X^{2+}$ have also been thoroughly examined experimentally in the Faraday geometry for an external magnetic field applied in the growth direction [111]. The measurements were performed with the field strength ranging in unit steps from $B=1$ up to $B=5 \mathrm{~T}$, and the luminescence was analyzed for the two different circular polarizations $\sigma^{+}$and $\sigma^{-}$. Under low power excitation, only four peaks associated with $X^{2+}$ can be resolved at $B=1 \mathrm{~T}$ [see Fig. 9(a)], but all six peaks expected from the numerical computations are clearly revealed in the experimental data at increased excitation power [see the four low-energy components in the spectra at the top of Fig. 9(b)]. The two weak-intensity components decrease with the magnetic field, and for $B=2 \mathrm{~T}$, their relative intensity is about $10 \%$ with respect to the dominating components, and they are not resolved at all for $B \geqslant 3 \mathrm{~T}$, leaving four components of $X^{2+}$, in agreement
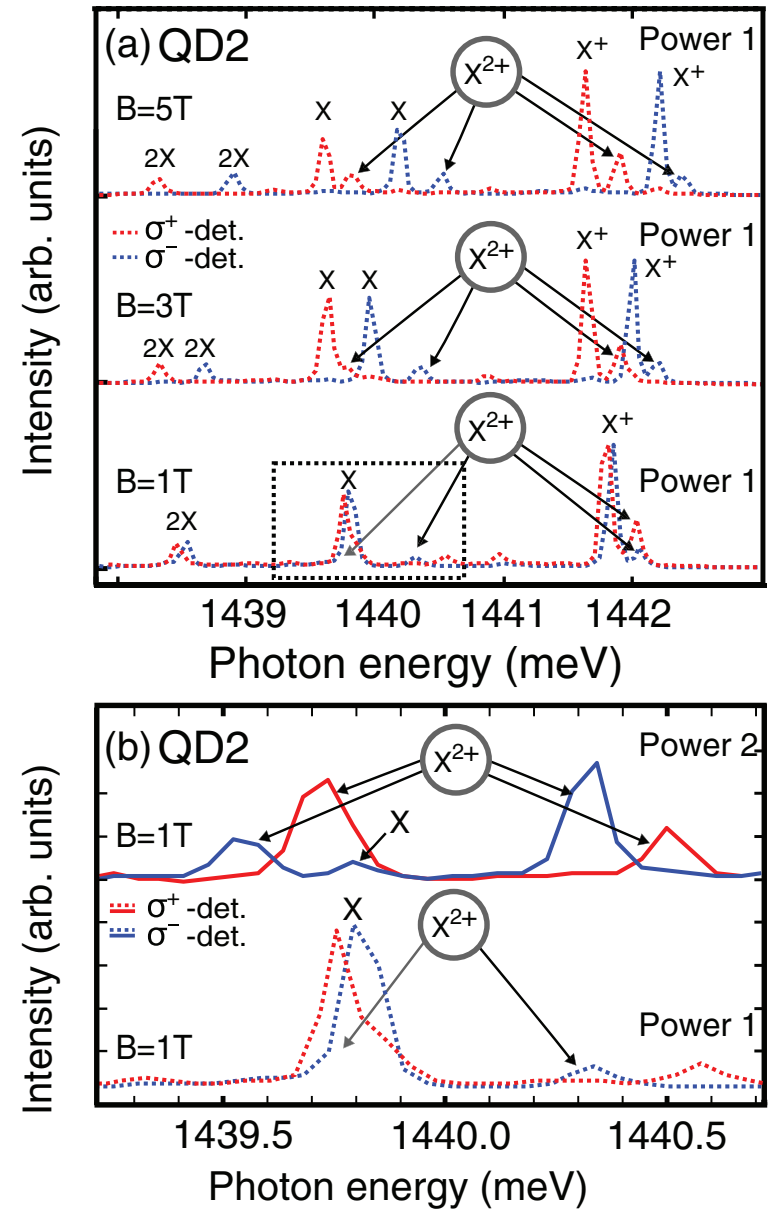

FIG. 9. (Color online) (a) Measured normalized polarizationresolved magnetic field spectra, from the same QD (QD2) as in Fig. 2(a), at three different field strengths using the same excitation power (power 1). (b) A magnification of the marked area in (a) is shown (bottom) together with a spectrum achieved at the same magnetic field strength $(B=1 \mathrm{~T})$, but with an increased excitation power. In both panels, the position for the components of the doubly positively charged exciton $X^{2+}$ is indicated by arrows.

with the numerical computations and consistent with the interpretation that an increased field strength restores the symmetry of the QD. The energy of each resolved spectral component is plotted as a function of the magnetic field strength, according to the dominating polarization helicity for QD2. In Fig. 10, the sequence of the three zero-field peaks of $X^{2+}$ has been labeled $p_{1}, p_{2}$, and $p_{3}$, respectively, starting with the highest recombination energy (see Fig. 10). The splittings of the peaks $p_{2}$ and $p_{3}$, detectable only at weak fields, are, within the resolution, identical, which is in perfect agreement with the derived decay scheme for $C_{2}$ symmetry, which predicts identical splittings $\Delta A$ for both peaks (see Fig. 8). Furthermore, the decay scheme predicts that the energy separation between the $\sigma^{-}$component of $p_{2}$ and the $\sigma^{+}$ component of $p_{3}$ is determined by a change of $\Delta E_{2}-\Delta E_{11}$. The nearly constant separation of these peaks seen in the experiments suggests that the magnetic field dependencies of $\Delta E_{2}$ and $\Delta E_{11}$ are comparable. 


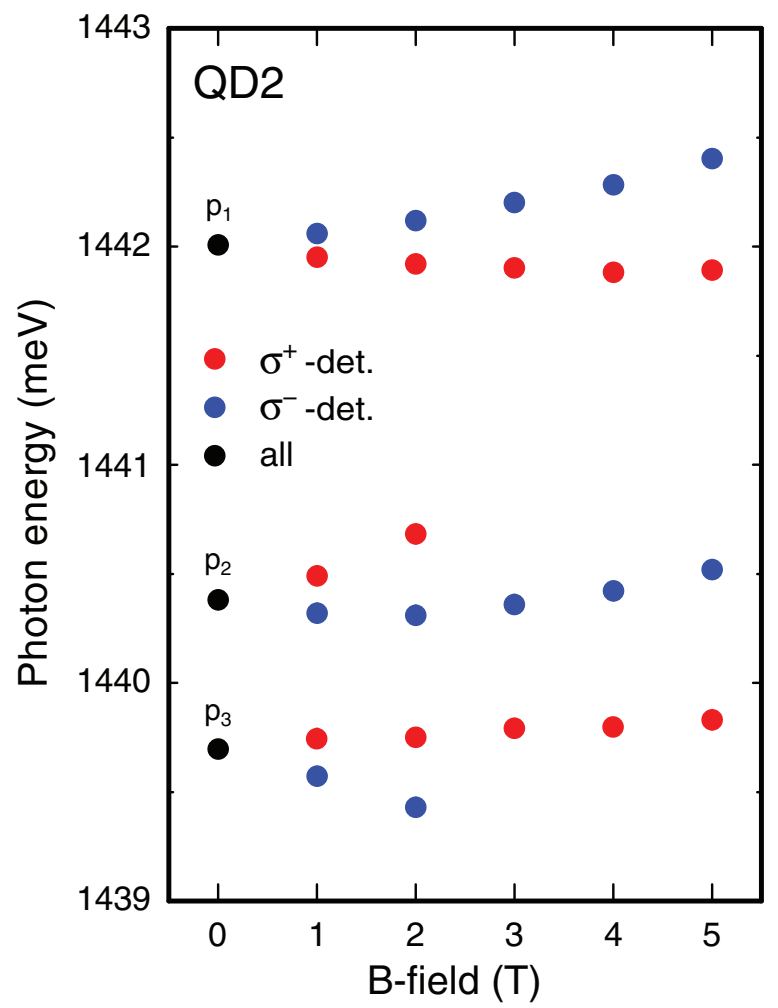

FIG. 10. (Color online) The measured energy for the components of the doubly charged exciton $X^{2+}$ plotted as a function of the magnetic field strength for the same QD (QD2) as in Figs. 2(a) and 4 .

\section{SUMMARY}

In summary, the doubly and triply positively charged excitons $X^{2+}$ and $X^{3+}$ were experimentally identified in the $\mu \mathrm{PL}$ spectra of pyramidal InGaAs QDs. The main spectral features of $X^{2+}$, determined by the two-hole final states of the recombination, provided information about the nature of the first excited hole level in the QD. It was concluded that the first excited hole level should be labeled $E_{1 / 2}$ for a QD belonging to point group $C_{3 v}$, and that this level is relatively isolated from the other excited hole levels. Moreover, it was demonstrated that the spectrum of $X^{2+}$ is very sensitive to QD asymmetry and that the experimentally measured QDs do exhibit a symmetry lower than the ideal $C_{3 v}$.

Our theoretical analysis demonstrated that no true triplet states exist for any two-hole system in a QD of symmetry $C_{3 v}$ or lower, although a singlet-tripletlike level arrangement may exist for quasi-degenerate excited levels and for an isolated excited level with label $E_{3 / 2}$. Instead, the two-hole system related to the final states of $X^{2+}$ for symmetric pyramidal QDs was predicted to exhibit a pair of true doublets, causing two well-resolved spectral peaks of comparable intensities. Only one of the two spectral peaks of $X^{2+}$ was found to split appreciably upon symmetry breaking, consistent with measurements of real QDs. Furthermore, the computational results as well as the group-theoretical analysis demonstrated a good agreement with experiments also when the QD is subjected to an external magnetic field. In conclusion, the doubly positively charged exciton is a good probe of QD asymmetry and could thus be used as a very efficient uninvasive postgrowth selection tool for locating highly symmetric QDs suitable for entangled photon emission.

\section{ACKNOWLEDGMENTS}

This research was enabled by the Irish Higher Education Authority Program for Research in Third Level Institutions (2007-2011) via the INSPIRE programme, Science Foundation Ireland under Grants No. 05/IN.1/I25 and No. 10/IN.1/I3000, grants from the Swedish Research Council (VR), and by equipment grants from the K. A. Wallenberg Foundation. We also acknowledge support from the Swedish Government Strategic Research Area in Materials Science on Functional Materials at Linköping University (Faculty Grant SFO-Mat-LiU No. 2009-00971). We are grateful to K. Thomas for his support with the MOVPE system.
${ }^{1}$ D. Gammon, Nature (London) 405, 899 (2000).

${ }^{2}$ A. Hartmann, Y. Ducommun, E. Kapon, U. Hohenester, and E. Molinari, Phys. Rev. Lett. 84, 5648 (2000).

${ }^{3}$ R. J. Warburton, C. Schaflein, D. Haft, F. Bickel, A. Lorke, K. Karrai, J. M. Garcia, W. Schoenfeld, and P. M. Petroff, Nature (London) 405, 926 (2000).

${ }^{4}$ K. V. Kavokin, Phys. Status Solidi A 195, 592 (2003).

${ }^{5}$ M. Ediger, G. Bester, B. D. Gerardot, A. Badolato, P. M. Petroff, K. Karrai, A. Zunger, and R. J. Warburton, Phys. Rev. Lett. 98, 036808 (2007).

${ }^{6}$ M. Ediger, G. Bester, A. Badolato, P. M. Petroff, K. Karrai, A. Zunger, and R. J. Warburton, Nature Phys. 3, 774 (2007).

${ }^{7}$ K. F. Karlsson, M. A. Dupertuis, D. Y. Oberli, E. Pelucchi, A. Rudra, P. O. Holtz, and E. Kapon, Phys. Rev. B 81, 161307 (2010).

${ }^{8}$ G. Juska, V. Dimastrodonato, L. O. Mereni, A. Gocalinska, and E. Pelucchi, Nature Phot. (2013), doi: 10.1038/nphoton.2013.128.
${ }^{9}$ A. Carmele, F. Milde, M.-R. Dachner, M. B. Harouni, R. Roknizadeh, M. Richter, and A. Knorr, Phys. Rev. B 81, 195319 (2010).

${ }^{10}$ E. Pelucchi, N. Moret, B. Dwir, D. Y. Oberli, A. Rudra, N. Gogneau, A. Kumar, E. Kapon, E. Levy, and A. Palevski, J. Appl. Phys. 99, 093515 (2006)

${ }^{11}$ V. Dimastrodonato, L. Mereni, R. Young, and E. Pelucchi, J. Cryst. Growth 312, 3057 (2010).

${ }^{12}$ E. Pelucchi, V. Dimastrodonato, A. Rudra, K. Leifer, E. Kapon, L. Bethke, P. A. Zestanakis, and D. D. Vvedensky, Phys. Rev. B 83, 205409 (2011)

${ }^{13}$ V. Dimastrodonato, E. Pelucchi, and D. D. Vvedensky, Phys. Rev. Lett. 108, 256102 (2012).

${ }^{14}$ A. Hartmann, Y. Ducommun, K. Leifer, and E. Kapon, J. Phys.: Condens. Matter 11, 5901 (1999).

${ }^{15} \mathrm{~V}$. Dimastrodonato, L. O. Mereni, R. J. Young, and E. Pelucchi, Phys. Status Solidi B 247, 1862 (2010). 
${ }^{16}$ M. H. Baier, A. Malko, E. Pelucchi, D. Y. Oberli, and E. Kapon, Phys. Rev. B 73, 205321 (2006).

${ }^{17}$ S. Altmann and P. Herzig, Point-group Theory Tables (Oxford Science, Clarendon, Oxford, 1994).

${ }^{18}$ M. A. Dupertuis, K. F. Karlsson, D. Y. Oberli, E. Pelucchi, A. Rudra, P. O. Holtz, and E. Kapon, Phys. Rev. Lett. 107, 127403 (2011).

${ }^{19}$ D. Dufåker, K. F. Karlsson, V. Dimastrodonato, L. O. Mereni, B. E. Sernelius, P. O. Holtz, and E. Pelucchi, Phys. Rev. B 82, 205421 (2010).

${ }^{20}$ S. P. Łepkowski, Phys. Rev. B 77, 155327 (2008).
${ }^{21}$ K. F. Karlsson, V. Troncale, D. Y. Oberli, A. Malko, E. Pelucchi, A. Rudra, and E. Kapon, Appl. Phys. Lett. 89, 251113 (2006).

${ }^{22}$ A. Barenco and M. A. Dupertuis, Phys. Rev. B 52, 2766 (1995).

${ }^{23}$ S. Healy, R. Young, L. Mereni, V. Dimastrodonato, E. Pelucchi, and E. O. Reilly, Physica E 42, 2761 (2010).

${ }^{24}$ It should be noted that signatures of symmetry also have been observed in other spectral patterns in Refs. 7, 18, and 8, even if no explanation was given in Ref. 8.

${ }^{25}$ T. Andlauer, R. Morschl, and P. Vogl, Phys. Rev. B 78, 075317 (2008).

${ }^{26}$ C. E. Pryor and M. E. Flatté, Phys. Rev. Lett. 96, 026804 (2006). 